\title{
Covid19 : The Specs from Immunity
}

Immunity is an important term directly related to prevent any kind of infection spreading particularly Covid19 under this pandemic situation . The specs of immunity depends on various factors like Foods \& Diets, Environment / Ecology , Good Habits, Natural Remedies like Herbs \& Anti-Oxidant therapy. Now we will discuss the every aspects of Immunity for boosting it up for a natural prevention of Covid19 ! . If we maintain the following factors strictly our immune system will be strong enough to fight against this Viral Curse!!

The most important is the REFERENCE section where some queries / discussions are pointed out related to the Immunity factors majorly .

Keywords : Lifestyle, Herd Immunity ,Natural Immunity, vaccination.

\section{Balanced and hygienic Diet -}

As per nutritional biochemistry a balanced diet is nothing but a appropriate combination of Protein, Carbohydrate \& Fats . Intake of animal \& vegetable protein is a matter of our interest . An healthy as well as healthy drinks should be an additional interest. The Diet factor also includes a great issue ie malnutrition - As we are all aware that Malnutrition is a great curse even in $21^{\text {st }}$ century due to Poverty, under-nutrition as well as heavy intake of fast foods causing some nutrition related disorders like Rickets, Scurvy , \& musculoskeletal disorders like Rh. Arthritis even neurological disorders this type of patients are in high risk zone in spreading the Corona Virus . The second component of Food is adulteration - it is a fact against the above three phenomenon as well as deterioration of Health even death . Adulteration is a great curse of Society by some intoxification of harmful chemicals as well as pathogenic contaminants . This is probably the greatest enemy of Immunity ie indirectly a friend of Corona attack . 
2. Anti-Oxidant / Therapeutic Anti-Oxidant - It include Balanced diet combined with Health Supplements, Herbs , Green Vegetables and Citrus food . The most important activity of antioxidant is to prevent the attack of Corona Virus as well as spreading of the Infection by quenching free radical also known as free radical scavenger. Green Tea as well as Black Coffee within specified limits helps the Patient for quick recovery.

3. Good Habits \& Wellness - Smoking damages the lungs through a coating of nicotine directly and the Patient is rapidly near the death by Pneumonic attack followed by sepsis ie death . The key point of wellness is to maintain a good mental health to relief stress during prolonged Lockdown period / under Quarantine condition . Sound SLEEP is a good secret of wellness ie to minimize the recovery time during attack of Covid19 .

4. Environment \& Ecology - Now we will look back the most important factor the SUSTAINABILITY of Environment or Ecology ie probably the major cause of the spreading of the virus through the weak Immune system! . The excessive stress of GREEN HOUSE GASES, OZONE HOLE - damage through UV attack, Heavy metal pollutants like lead ( $\mathrm{Pb})$, Arsenic in waste water as well as air pollutants . Higher SUSPENDED PARTICULATE MATTER ( SPM ) content will spread this infection through aerosolised condition (https://www.researchgate.net/post/Do_We_belive_that_spreading_of_COVIDinfection_is_an_example_of_aerosol_type_of_Colloid ).

\section{SELF REFERENCENCE FOR DETAIL ( Datta J , Researchgate.net ) :}

1. https://www.researchgate.net/post/Whether successive vaccination will increase or decrease HERD_IMMUNITY .

2. https://www.researchgate.net/post/Time_is_the_ultimate_remedies_from_Covid_variantsNothing_else.

3. https://www.researchgate.net/post/Herbs-medicinal_use .

4. https://www.researchgate.net/post/Covid19 Vaccination-Inactive vaccination.

Shall we beleive that the Covid19 has a asymmetric characteristics of Malaria and Crown virus ? (Started May 1 ).

What is Malnutrition - Do You agree it is a Global Problem particularly in the Third world countries ? ( Asked

December 11,2019) .

What is GREEN HEALTH ? ( Asked December 1, 2019).

What is Food adulteration - Is it a Big Problem ? (Asked November 11, 2019)

What is balanced diet - Will You support that it is essential to fight agaist COVID-19 ? (Asked November 7,2019 ). 
What do you prefer to take ANIMAL or VEGETABLE protein under attack of corona virus ? ( Asked October 28,2019 ).

Mental Frustation during LOCKDOWN period of Covid19 is more prone towards infection Share your feelings ? ( Asked December 19,2019 ).

What is SLEEP ? ( Asked October 9,2019 )

Your favourite food as Anti-Oxidant - Let us discuss ? ( Started September 27 , 2019 )

TEA or COFFEE - Which one is good for heath ? ( Started October , 29,2019 )

1. Jaydip Datta , HEALTH \& ENVIRONMENT: A SOCIOLOGICAL CONCIOUSNESS ,November 2019 ,

DOI: $\underline{10.13140 / R G .2 .2 .23247 .84646 / 2}$,License ,CC BY-SA 4.0

(

https://www.researchgate.net/publication/337480620 HEALTH ENVIRONMENT A SOCIOLOGICAL CON CIOUSNESS? sg=1XNKtRO wgA3S6WkAeJG5-

vD0NiY1YyaMEC9MJzluL0CBnuOc1L0AEm24vcDvR0cd9qi fijubDgCPDMaLYec9nJjova6VmM5vYkXB7X.

5wMwvF bh3GkLHNmi9ywovP90tV-

6NNrRaLtAwMqLwzBPe0J0SxgmaVC5wBj21MSgq3Jdkfp12cGi5Dk36IK9A )

2. Jaydip Datta , BALANCED DIET \& ADULTERATION : An Impression,February 2020

,DOI: $10.31219 / \mathrm{osf} . \mathrm{io} / \mathrm{n} 3 \mathrm{cbp}$.

(

(https://www.researchgate.net/publication/339412741 BALANCED DIET ADULTERATION An Impression ? sg=4ft-eYeqHecOfxTnXMTbJpMIEvvCdj6MNvjCkxvGC7F5GsA-Bedzu4TT-

zf8BlqvOl6l71 XXqwwqCkOilCTdZTAljVI2JW6olZbR9H8.Khy0QuXfMocAWfAtr7JIr8vJleCix0n LIADDLMr5 ni-DidO92PhGDwENI0aCddHttp9T3H2EOIU4Pr4QkYOkw )

3.Jaydip Datta , COVID ( 2020-22)Awareness Control Programme: Sustainability of Health And Environment ,April 2022 ,DOI: 10.31219/osf.io/agqj4 .

DATTA, J. (2021, January 2). COVID ( 2020-22 )Awareness Control Programme: Brief Review.

https://doi.org/10.31219/osf.io/agqj4

DATTA, JAYDIP. “COVID ( 2020-22 )awareness Control Programme: Brief Review.” OSF Preprints, 2 Jan. 2021.

Web.

DATTA, JAYDIP. 2021. “COVID ( 2020-22 )awareness Control Programme: Brief Review.” OSF Preprints. January 2. doi:10.31219/osf.io/agqj4.

(https://www.researchgate.net/publication/348179565 COVID 202022Awareness Control Programme Sustainability of Health And Environment? sg\%5B0\%5D =8M-

AOKP hyPUeEFnycvcA5keujYsHzNBE9VReQ 2USZs2n1Gpca 7fF7JmORUH feKTcTMGA0TdQ36613 JBsdxmCTBK6u5w2DUmKSDz2.M3YA8eG sZbUFbQszb0hj2zQuSrmfLtr1JvrmBCoifjdhr8GVLnj8JTwug HPewjMwS2hyC17RrEci19ggy00JQ).

4.Jaydip Datta, PSYCHOLOGICAL IMPACT: AN IMPRESSION THROUGH QUERIES ,February 2020 ,DOI: 10.13140/RG.2.2.20707.89120/1 .

(https://www.researchgate.net/publication/338987183 PSYCHOLOGICAL IMPACT AN IMPRESSION TH ROUGH QUERIES? sg= IRYwgRZNvGfgFylZgv6pWcoWmr04zTlh-

PifrBRY7xzoOrXCwt7P3pkAExA07dgp92YiR33WY3|3ixMqtKqrPGeBNIBnGGqhLhYvv7t.KYfHVq510UBI2a eWkJOQ8eyKQ7YBI9RsSXZx4ZZdalBsGyuTpGVDh eUeF6F6q6qKYal5yS6XUW2dHQ7zFnHgQ )

5. Jaydip Datta ,Therapeutic Review on Aloe Vera - A Traditional Phytomedicine ,February 2013,DOI: $\underline{10.6084 / \mathrm{m} 9 . \text { figshare.12179874.v1 . }}$. 
(https://www.researchgate.net/publication/335995031 Therapeutic Review on Aloe Vera -

A Traditional Phytomedicine? sg\%5B0\%5D=aEihifiZIHt8jCK8gXystnczSFh7eUZgaWZ96X gq32BQ8B h7JJ5PdwVeP0-UDyQJ13jtVBkhgDfpwtk-rEBiu92k57lhyRA-

PvAYW8YT.oZb06FT2L6JVLYGh32jdtyYEsZcsJxViekPCP-

dFVPZRAgl15IDDBborihWmpkO7141HDCyHmUlrT9-3ljd3rg ).

DATTA, JAYDIP (2020): Therapeutic Review on Aloe Vera - A Traditional Phytomedicine.

figshare. Journal contribution. https://doi.org/10.6084/m9.figshare.12179874.v1 